\title{
Enabling a Paradigm Shift in CryoEM Sample Preparation with chameleon
}

\author{
Michele C. Darrow, Tim Booth, John P. Moore, Klaus Doering, Paul Thaw and Russell S. King
}

SPT Labtech LTD., Melbourn Science Park, Hertfordshire, UK, United States

The period responsible for the current generation of cryogenic electron microscopy (cryo-EM) instrumentation and image processing software is often referred to as the "resolution revolution." The results of these technological advancements have been achievements in higher quality structures and cryo-EM becoming the go-to method for structural biologists. However, sample preparation is widely recognised as a key unresolved step in the high-resolution cryo electron microscopy (cryoEM) workflow [1].

While it has been demonstrated that conventional plunge-freezing can achieve atomic resolution on the latest electron microscopes with ideal specimens, real world results still struggle to break the resolution barriers needed to answer the detailed biological questions at hand. The one size fits all approach of traditional blot and plunge freezing methods does little to address sample specific characteristics and reduce variability. The resulting inconsistent ice quality and unmitigated air water interface $(\mathrm{AWI})$ effects lead to unnecessary screening along with experimental repetition with untraceable results.

To keep pace with the rapidly advancing hardware and software capabilities, next generation sample preparation methods have been realized through emerging instruments such as the chameleon to minimize lengthy sample optimization workflows and enable routine high-resolution reconstructions. Bringing together robotics, automation, and intelligent software allows experts and novice users alike quicker access to consistent results [2]. Users can confidently work with the instrument to control the freezing process with guided software protocols intended to handle the experimental framework and limit manual intervention.

The chameleon achieves this automation through the novel design of picolitre dispensing onto selfwicking nanowire grids [3-4]. Reducing the deposited sample volume and eliminating the blotting step allows for an advanced degree of control over the thin film formation step and subsequent vitrification quality, while still enabling rapid dispense-to-plunge times as fast as $54 \mathrm{~ms}$. Recent experiments demonstrate that AWI effects are ubiquitous and sample specific [5-7]. It has been shown that reducing interactions with the harmful AWI can have beneficial effects, improving data quality and therefore reducing the amount of data necessary to achieve high-resolution reconstructions [4-6, 8-9].

Incorporating chameleon into the cryo-EM workflow represents a paradigm shift in the approach to the bottleneck of specimen optimization and screening. To reduce the number of research cycles necessary to define a clear path to success, the first step should be to achieve reproducible highquality specimens. Using the chameleon unlocks a researcher's ability to vary dispense-to-plunge times to meet individual specimens' needs, providing understanding of unique specimen characteristics and behaviours, and a path to mitigating the negative effects often seen during traditional blot-freezing of specimens with these characteristics. Recent results utilising variable dispense-to-plunge times and the effects of doing so will be presented for a variety of specimens.

The chameleon also reduces the knock-on effects caused by poor sample preparation. The screening load on microscopes is reduced by allowing researchers to control and then screen for film thickness using onboard cameras during the sample vitrification process. During the expensive 
cryo-EM screening step, loading specimens with known ice quality can further reduce the time and financial costs associated with searching many grids with inconsistent, non-uniform ice quality on each. This optimizable vitrification approach can be used to capture unique sample behaviour and can reduce the time and costs associated with trial-and-error experimental optimization necessary using traditional methods [10].

The literature demonstrates the clear benefits of chameleon as a platform for optimization when determining high resolution structures of samples suffering from AWI denaturation or preferred orientation and when working with samples suffering from inconsistent ice outcomes with traditional freezing methods. In addition, the democratisation of the sample preparation process through automation and ease-of-use improvements provides a path for new researchers to successfully adopt cryoEM.

\section{References}

1. B. Carragher, Y. Cheng, A. Frost, R.M. Glaeser, G.C. Lander, E. Nogales, H.-W. Wang, Current outcomes when optimizing 'standard' sample preparation for single-particle cryo-EM Journal of Microscopy, 276(1), pp.39-45.

2. Darrow, M. C., Moore, J. P., Walker, R. J., Doering, K. and King, R. S. (2019) "Chameleon: Next Generation Sample Preparation for CryoEM based on Spotiton," Microscopy and Microanalysis. Cambridge University Press, 25(S2), pp. 994-995.

3. Dandey, V.P., Wei, H., Zhang, Z., Tan, Y.Z., Acharya, P., Eng, E.T., Rice, W.J., Kahn, P.A., Potter, C.S., Carragher, B., 2018. Spotiton: New Features and Applications. J Struct Biol 202, 161-169.

4. Wei, H., Dandey, V.P., Zhang, Z., Raczkowski, A., Rice, W.J., Carragher, B., Potter, C.S., 2018. Optimizing "self-wicking" nanowire grids. Journal of Structural Biology 202, 170-174.

5. Noble, Alex J., Wei, H., Dandey, V.P., Zhang, Z., Tan, Y.Z., Potter, C.S., Carragher, B., 2018. Reducing effects of particle adsorption to the air-water interface in cryo-EM. Nat Methods 15, 793795.

6. Klebl, D.P., et al., 2020. Need for speed: examining protein behavior during CryoEM grid preparation at different timescales. Structure, 28(11), pp.1238-1248.

7. Glaeser, R.M., 2018. PROTEINS, INTERFACES, AND CRYO-EM GRIDS. Curr Opin Colloid Interface Sci 34, 1-8.

8. Liu, Y., Zhou, K., Zhang, N., Wei, H., Tan, Y.Z., Zhang, Z., Carragher, B., Potter, C.S., D'Arcy, S. and Luger, K., 2020. FACT caught in the act of manipulating the nucleosome. Nature, 577(7790), pp.426-431.

9. Liang, W., Wei, H., Noble, A., Mo, S., Lee, D., Mancl, J., King, J.L., Pan, M., Koehler, C., Zhao, M. and Potter, C., 2020. Structural basis for the mechanisms of human presequence protease conformational switch and substrate recognition.

10. Huo, J., et al., 2020. Neutralizing nanobodies bind SARS-CoV-2 spike RBD and block interaction with ACE2. Nature structural \& molecular biology, 27(9), pp.846-854. 